\title{
Nésoblastome et ulcère gastro-duodénal
}

Aucune explication satisfaisante n'avait été apportée à la pathogénie de ces ulcères éternellement récidivants, malgré une thérapeutique chirurgicale de plus en plus mutilante, jusqu'aux de -R. M. Zollinger et E. H. Ellison (Ann. Surg., vol. 142, p. 709, oct. 1955) de Colombus (Ohio): pour ces auteurs, l'association d'une tumeur des îlots de Langerhans avec un ulcère, signalée dans quelques observations éparses, n'est pas fortuite. L'année suivante, Ellison pouvait déjà dénombrer 24 cas (d'ailleurs pas tous authentiques) de cette association, à laquelle Eísenmann et Maynard (Gastroenterology, vol. 31, p. 296, sept. 1956) proposèrent de conférer le nom de «Syn-drome de Zollinger-Ellíson»; au Congrès international de Washington, en mai 1958, Zollinger en annonçait 75 observations publiées.

Ce syndrome est défini par la triade suivante:

Ulcères, fréquemment multiples ( $1 / 4$ des cas), de sieges très souvent atypiques: sur 30 lesions découvertes chez les 22 premiers malades, 9 étaient jéjunaux, 5 sur les 2 e et $3 \mathrm{e}$ portions du duodenum, un æsophagien, soit 17 atypiques; et d'une particulière gravité, à cause des complications inhérentes aux inevitables récidives. Ces ulcères résistent non seulement aux traitements médicaux les plus assidus et à la radiothérapie, mais encore à la vagotomie sus- et sous-diaphrag-matique et à la gastrectomie subtotale, la lesion se reproduisant tant qu'il existe tant soit peu de muqueuse gastrique.

Hypersécrétíon gastrique massive: le volume produit pendant les 12 heures d'une nuit dépasse toujours $1000 \mathrm{ml}$. (au lieu des $600 \mathrm{ml}$. normaux) et atteint parfois 3 litres; ce sue gastrique contient jusqu'à $600 \mathrm{mEq}$. d'acide chlorhydrique (au lieu de $20 \mathrm{mEq}$.) secretes pendant la même période. Aussi Ellison propose-t-il (Surgery, vol. 40, p. 147 [1956]) qu'un nésoblastome* soit suspecté chaque foís que le sue gastrique des 12 heures nocturnes dépasse 2 litres et $100 \mathrm{mEq}$. Hcl. De plus,

* Nésoblastome $=$ tumeur des îlots de Langerhans (et non «nésidioblastome» comme cela a été

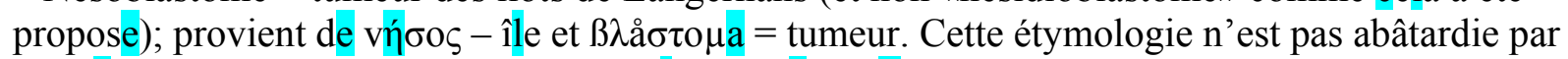
un melange de latin et de grec (comme «insulome»); elle désigne les néoplasies

Langerhansiennes de toute espèce, sans pré-juger de leur nature, insulome étant reserve aux proliferations des cellules- $\beta$ avec hypersécrétion d'insuline.

188

Editorial

selon Wadell et coll. (New Engl. J. Med., vol. 260, p. 56 [1959]) les stimulants habituels de la secretion gastrique (insuline, histamine) dépriment celle-ci au lieu de l'augmenter.

3. Tumeur des îlots de Langerhans, non ínsulíníenne; elle ne com-porte pas de cellules- $\beta$ et n'entraîne par consequent pas d'hypogly-cémie. Deux fois sur trois, la tumeur est maligne et propagée hors des limites du pancreas (foie, ganglions, etc.); parfois, en Гabsence d'une localisation repérable, il s'agissait d'une adénomatose diffuse du pancreas. *

A juste titre, Zollinger et Ellison émirent l'hypothèse d'un facteur ulcérogène humoral à point de depart pancréatique. Quand on derive la secretion externe du pancreas (chez le chien), il se 
produit eonstamment un ulcère, par manque de neutralisation alcaline; mais si l'on procède à une pancréatectomie totale, supprimant du même coup les secretions externe et interne, $\Gamma$ ulcère se développe à peine chez $2 \%$ des chiens; la presence du pancreas est done indispensable à la production de l'ulcère.

L'insuline possède un effet hypersécrétant sur Гestomac, par Гinter-médiaire du vague, avec hypoglycémie concomittante; et il existe plusieurs cas dHnsulome associé à Гulcère gastroduodénal, colligés par Leffkowitz et al. (Gastroenterologia, vol. 93, p. 157, mars 1960). Aussi s'étonne-t-on de voir incriminer le glucagon, qui possède une action antagoniste à celle de l'insuline; d'après Spíro et al. ( $5^{\circ}$ Congr. Europ. de Gastro-entérologíe, Leyden, avril 1960), une injection continue de glucagon provoque 1 'apparition d'une achylie durable.

A côté des cellules- $\alpha$ et $-\beta$, productrices respectivement du glucagon et de l'insuline, il existe encore dans le pancreas des cellules- $\gamma$ et $-\delta$ dont le role physiologique et $\Gamma$ hormone sontinconnus. Nous ensommes done réduits à postuler Texistence d'un facteure ulcérogène d'Origine pancréatique, transmis vraisemblablement par voie humorale (puis-que la vagotomie n'en empêche pas Гactivité) et determinant des lesions récidivantes quand il est produit en quantité excessive par un nésoblastome.

Avant la découverte de Torigine pancréatique de ces ulcères récidi-vants, le traitement chirurgical était des plus décevants. Parmi les 22 premiers cas publiés, deux ne furent pas opérés et moururent de complication ulcéreuse: 6 autres subirent une seule operation, les 14 resEditorial

189

tants de deux à cinq interventions itératives; la tumeur pancréatique fut découverte (tumeur palpable) 2 fois avant toute operation, 3 fois au cours-même de la laparotomie, 11 fois à l'autopsie seulement; seuls survécurent ceux dont le nésoblastome fut réséqué.

Dans une publication récente (Gastroenterology, vol. 37, p. 401, oct. 1959) Zollínger avec D. W. Elliott condensent leur attitude thé-rapeutique comme suit:

Aucun test de laboratoire ne permettant de prouver l'existence d'un adénome ulcérogène du pancreas, il faut le suspecter systêmati-quement en presence d'une localisation inhabituelle (surtout jéjunale) de l'ulcère, ou quand un ulcère récidive peu de temps après une intervention chirurgicale adequate.

Si l'on a une bonne raison d'admettre l'existence d'un adénome ulcérogène, et que Гexploration n'en trouve pas à la laparotomie, il faut réséquer le corps et la queue du pancreas: une atteinte hyper-plasique des îlots de Langerhans sera souvent mise en evidence, et l'opération sanctionnée par 1'extinction de la maladie ulcéreuse.

Si l'on trouve le nésoblastome prévu, on doit pratiquer une ... gastrectomie totale! Cette conclusion thérapeutique paradoxale est dictée par Гincertitude d'enlever tout le tissu à fonction ulcérogène, soit qu'il existe une adénomatose diffuse de la glande, soit que des métastases continuent à sécréter après une pancréatectomie totale: il convient done d'enlever toute muqueuse capable de produire de l'HCl.

*

L'intérêt des influences hormonales susceptibles de déclencher la maladie ulcéreuse ou d'en entretenir la chronicité ne se limite pas aux relations pancréas-estomac.

L'effet néfaste sur l'estomac des cortico-stéroïdes en représente Гexemple le plus frappant à Theure actuelle; maisle role nuisible des secretions thyroïdienne, parathyroïdienne et insulinienne égale-ment reconnu, Tinfluence favorable de la DOCA et des hormones sexuelles, en revanche, utilisée en thérapeutique. 
Dans le syndrome de Zollínger-Ellison, on a découvert souvent d'autres atteintes glandulaires; sur les 75 cas colligés par son auteur, 15 malades étaient porteurs d' 'adénomes multiples: au total 38 lesions, dont 10 cortico-surrénaliennes, 7 hypophysaires et 5 parathyroïdiennes! Zollinger ajoute que l'existence d'un facteur hypersécrétoire endocrinien chez un ulcéreux est un argument de plus pour rechercher la coexistence d'un nésoblastome. M. D. 